\title{
Des pierres pour Saturne. Une relecture des Mémoires des Girondins
}

Des pierres pour Saturne. A Reevaluation of the Memoires of the Girondins

\section{Anne de Mathan}

\section{(2) OpenEdition \\ 12 Journals}

\section{Édition électronique}

URL : https://journals.openedition.org/ahrf/12883

DOI : $10.4000 /$ ahrf. 12883

ISSN : 1952-403X

Éditeur :

Armand Colin, Société des études robespierristes

\section{Édition imprimée}

Date de publication : 1 septembre 2013

Pagination : 165-188

ISBN : 9782200928261

ISSN : 0003-4436

Référence électronique

Anne de Mathan, « Des pierres pour Saturne. Une relecture des Mémoires des Girondins », Annales historiques de la Révolution française [En ligne], 373 I juillet-septembre 2013, mis en ligne le 01

septembre 2016, consulté le 01 juillet 2021. URL : http://journals.openedition.org/ahrf/12883 ; DOI : https://doi.org/10.4000/ahrf.12883 


\title{
DES PIERRES POUR SATURNE. UNE RELECTURE DES MÉMOIRES DES GIRONDINS
}

\author{
Anne DE MATHAN
}

\begin{abstract}
La relecture critique des neuf Mémoires rédigés par des girondins en prison (Brissot et Mme Roland) ou en fuite (Buzot, Pétion, Barbaroux, Louvet, Isnard, Meillan et Riouffe) montre comment ces vaincus qui tentent de sauver leur honneur, présentent et se représentent leur rôle dans la Révolution, les causes du conflit avec la Montagne et de leur défaite, leurs épreuves intimes. Marquées par l'héritage classique, les Lumières et Rousseau, ces confessions révèlent la cohésion d'une culture commune, moins différente de celle des Montagnards qu'on ne l'a dit. Défense et illustration des girondins, ces textes de combat stigmatisent leurs adversaires dont ils prétendent démasquer les coupables projets. Récits autobiographiques décrivant les souffrances de la réclusion ou de la proscription, ils scénarisent la force de l'ipséité face à la perspective de la mort et confèrent une dimension romantique aux destinées des girondins qui espèrent agir sur leur postérité dans l'histoire.
\end{abstract}

Mots-clés : girondins, mémoires, Terreur, an II.

Après l'exécution des girondins et de Mme Roland à Paris, ainsi que de nombreux fugitifs en province, François Buzot tente, en souvenir de Vergniaud qui redoutait que « la Révolution, comme Saturne dévorant successivement tous ses enfants, n'engendrât enfin le despotisme ${ }^{1}$, de donner telle Cybèle des pierres à Saturne afin de résister à l'opprobre et l'oubli :

(1) AP, LX, 13 mars 1793, p. 161-166. 
«Honorables victimes de la tyrannie, vous serez vengées! Un jour la postérité ne prononcera vos noms qu'avec le recueillement de la vénération et de la reconnaissance. Vous êtes morts, comme Phocion et Sidney, pour la liberté de votre pays ; comme eux, vous vivrez dans la mémoire des hommes de bien. L'ami de la sagesse et de l'humanité viendra méditer sur votre tombe vos leçons et ses droits $»^{2}$.

Cette invocation dit bien l'espoir que l'échec politique, l'infamie du décret d'arrestation, les affres de la clandestinité ou le sang versé sur l'échafaud seront un jour réparés en honneur.

Les textes rédigés par des girondins incarcérés - Brissot et Mme Roland - ou fugitifs - Buzot, Pétion, Barbaroux, Louvet, Isnard, Meillan et Riouffe - qui ressortissent aux confessions ultimes, tant qu'aux projets de défense et parfois aux pamphlets thermidoriens, constituent une belle source à retravailler à l' aune des renouvellements historiographiques sur les documents autobiographiques ${ }^{3}$, comme le proposait Michel Vovelle :

« Le temps est sans doute venu de revisiter attentivement ces témoignages personnels, non point tant pour y découvrir de l'inédit ou du nouveau sur une Révolution qui n'offre plus de grands mystères, mais pour ce qu'ils nous apportent sur la psychologie d'un acteur, d'un groupe [...] C'est un regard que nous cherchons à saisir, la façon dont [..] a été vécue, rêvée, reconstruite une expérience le plus souvent tragique, même lorsqu'elle ne conduit pas à la mort $»^{4}$.

La fiabilité de l'établissement de ces textes varie. À l'Abbaye, Brissot rédigea des Mémoires dont le manuscrit a disparu à une date inconnue. Les éditions de 1830 et 1877 ayant grossi le texte initial par des articles tirés du Patriote Français, celle de Claude Perroud a été préférée ${ }^{5}$. Les Notices historiques, des Portraits et anecdotes, et des Mémoires particuliers de

(2) Charles Dauban, Mémoires inédits de Pétion, et mémoires de Buzot et de Barbaroux, Paris, Plon, 1866, p. 98.

(3) Jean-Pierre BARDET et François-Joseph RUGGIU (dir.), Au plus près du secret des cœurs ? Nouvelles lectures historiques des écrits du for privé en Europe du XVI e au XVIII ${ }^{e}$ siècle, Paris, PUPS, 2005 ; Jean-Pierre BARDET, Élisabeth ARNOUL et François-Joseph RUGGIU, Les écrits du for privé en Europe, du Moyen Age à l'époque contemporaine. Enquêtes, analyses, publications, Bordeaux, PUB, 2010 ; Sylvie MouYSSET, Jean-Pierre BARDET, François-Joseph RUGGIU (dir.), "Car c'est moy que je peins ». Individu et liens sociaux dans les écrits du for privé en Europe, de la fin du Moyen Âge à 1914, Toulouse, Méridiennes, 2010.

(4) Jean-Baptiste LOUVET, Mémoires. Quelques notice pour l'histoire et le récit de mes périls depuis le 31 mai 1793, préface de Michel VovelLE, Paris, Desjonquères, 1988, p. II. Picard, 1911

(5) Jacques-Pierre BrISSOT, Mémoires, édition critique de Claude PERROUD, 2 volumes, Paris, 
Manon Roland ont été publiés en l'an III par Louvet sous le titre Appel à l'impartiale postérité. Champagneux en proposa une édition en 1799, plus complète et conforme, avant celle de 1820 chez Baudoin qui donna lieu à de nombreuses rééditions au $\mathrm{XIX}^{\mathrm{e}}$ siècle. Celle du Mercure de France comporte en outre des observations, des notes sur son procès et son projet de défense ${ }^{6}$. Buzot composa ses Mémoires entre l'exécution des girondins à Paris (30 octobre 1793) et le départ de Louvet (13 novembre) ; les notes et les ultimes passages ajoutés mi-juin 1794 attestent sa radicalisation politique. Saisis chez ses protecteurs, ses papiers furent adressés au comité de salut public, avec ceux de Pétion et Barbaroux remis à leurs descendants ; une copie de ceux de Buzot servit à l'édition de Julien Guadet, neveu du Conventionnel, en 1823. Celui-ci ayant retouché le texte, lui a été préférée l'édition plus fidèle de Charles Dauban avec les mémoires de Pétion en $1766^{7}$. Des Mémoires de Barbaroux, utilisées dans la version originale de $1822^{8}$, il ne reste qu'une partie, évoquant le début de la Révolution jusqu'à la Convention. Louvet rédigea et édita Quelques notices pour l'histoire et le récit de mes périls depuis le 31 mai 1793, qui connurent trois éditions en l'an III et des rééditions au XIX ${ }^{\mathrm{e}}$ siècle. Michel Vovelle en proposa une édition augmentée d'un manuscrit que Louvet n'avait pas retrouvé ${ }^{9}$. Isnard publia sa Proscription en l'an III $^{10}$, où Meillan rédigea aussi ses Mémoires d'un proscrit, mais l'édition posthume de 1823 a été reprise telle quelle lors du Bicentenaire ${ }^{11}$. Les Mémoires pour servir à l'histoire de la tyrannie de Robespierre par Riouffe eurent deux éditions en l'an III $^{12}$ reprises au $\mathrm{XIX}^{\mathrm{e}}$ siècle $^{13}$.

Il ne s'agit pas là d'instruire un procès en réhabilitation, ni de reconstituer les tribulations bien connues de ces vaincus, mais de contribuer à la construction d'une histoire dégagée des passions mémorielles en revisitant des sources encore peu utilisées ${ }^{14}$ afin d'observer comment les

(6) Mme Roland, Mémoires, édition Paul de Roux, Paris, Mercure de France [1966], 1986.

(7) Charles DAUBAN, op. cit.

(8) Charles BARBAROuX, Mémoires, édités par MM. Berville et Barrière, Paris, Baudoin,

1822.

(9) Michel VovelLe, op. cit.

(10) Maximin ISNARD, Proscription d'Isnard, Paris, Isnard, an III.

(11) Arnaud-Jean MeILlan, Mémoires d'un proscrit, rééd. Christian DesPlat, Bordeaux, Olivier Bervialle, 1989.

(12) Honoré RIOUfFE, Mémoires pour servir à l'histoire de la tyrannie de Robespierre, Paris, Anjubault, Mahé, Louvet, an III.

(13) Anne de Mathan, Histoires de Terreur. Les Mémoires de Armand Cholet et Honoré Riouffe, Paris, Honoré Champion, à paraître.

(14) Jean-Luc CHAPPEY, «La Révolution française dans l'ère du soupçon. L'enjeu des mémoires révolutionnaires », Cahiers d'Histoire. Revue d'Histoire critique, 1996, p. 63-76; Sergio LuZZATO, Mémoires de la Terreur. Vieux Montagnards et jeunes Républicains au XIXe siècle, Lyon, 
girondins tentèrent d'historiciser leur sort, et d'analyser ces présentations et représentations d'eux-mêmes et de leur rôle dans la Révolution, des causes du conflit avec la Montagne et de leur défaite, de leur destinée voire de leur mort. Ces mémoires d'outre Styx scénarisent l'ultime combat des girondins pour sublimer leurs maux en mots et tenter de sauver ce qui pouvait encore l'être : la postérité. Passés au crible d'une relecture critique, complémentaire des approches quantitatives - comptages ${ }^{15}$ et prosopographie $^{16}$-, ils révèlent la cohésion d'une culture commune. Ces textes de combat procèdent à une virulente stigmatisation des adversaires politiques et valorisent un dévouement sacrificiel à la chose publique. Récits autobiographiques, ils montrent dans les souffrances de la vie de reclus ou de proscrit, les ressources d'un moi préromantique face à la possibilité de la mort.

\section{Une culture commune}

Les Mémoires des girondins frappent par la cohérence d'une culture commune, marquée par une formation classique, le déisme plutôt que l'athéisme, les œuvres des Lumières et particulièrement Rousseau qu'ils célèbrent et imitent à l'envi.

Mme Roland, se targuant de passions républicaines formées dès l'enfance, revendique idéaux et valeurs des grands hommes de l'Antiquité :

« Je soupirais en songeant à Athènes où j' aurais également admiré les beaux arts [....], je me promenais en esprit dans la Grèce, j'assistais aux jeux olympiques et je me dépitais de me trouver française. Ainsi frappée de tout ce que m'avait offert le beau temps des républiques, je glissais sur les orages dont elles avaient été agitées ; j'oubliais la mort de Socrate, l'exil d'Aristide, la condamnation de Phocion. Je ne savais pas que le ciel me réservait pour être témoin d'erreurs pareilles à celles dont ils furent les victimes et participer à la gloire d'une persécution du même genre, après avoir professé leurs principes ».

PUL, 1988 ; Natalie PETITEAU, Écrire la Révolution. Les mémorialistes de la Révolution et de l'Empire, Paris, Les Indes savantes, 2012.

(15) Voir les travaux de Michael John Sydenham, Alison Patrick, Jacqueline Chaumié, Patrice Higonnet, Michael LeVIS-BeCK, Anne HiLdRETH et Alan SPITZER.

(16) Anne de MATHAN, Girondins jusqu'au tombeau. Une révolte bordelaise dans la Révolution, Bordeaux, Sud-Ouest Éditions, 2004. 
Louant la bonne volonté de ses parents à étancher sa soif d'apprendre, elle marque la distinction culturelle : "Mon père, n'étant pas dans le cas de choisir, demandait ce que je lui indiquais $»^{17}$.

Brissot conte aussi un goût précoce pour la république :

« J'ai détesté les rois de bien bonne heure [...] Cette idée de révolution, que je n'osais avouer, roulait souvent dans ma tête ; je m'y donnais un des rôles principaux, comme il est bien naturel de le croire. L'histoire de Charles $1^{\text {er }}$ et de Cromwell m'avait singulièrement frappé, je me rappelais sans cesse ce dernier, déchirant, dans son enfance, le portrait de son roi, terminant sa carrière par le faire décapiter, et ne devant qu'à son génie le grand rôle qu'il avait joué dans la révolution anglaise. Il ne me paraissait pas impossible de renouveler cette révolution ».

Se posant en homme des Lumières, il évoque la modestie de son ascendance pour oser des comparaisons flatteuses : «Si c'est un sujet de plaisanterie d'être né dans la classe des artisans, moi chétif j'y suis exposé avec les plus beaux génies. Théophraste, Horace, Virgile, Massillon, Diderot, Franklin, les deux Rousseau sortaient de cette classe de la société». Il recherche l'empathie du lecteur tout en se classant culturellement : « Même au milieu des succès de mon éducation, lorsque je revenais la tête chargée des lauriers du collège, mon père ne m'embrassait qu'avec une sécheresse qui pénétrait jusqu'à mon cœur et le resserrait. [...] Du moment où je devins instruit, il me sembla que je leur devenais étranger $»^{18}$.

Isnard place en exergue une maxime de Solon et récite ses héros romains du collège, afin de transmuer le proscrit en un citoyen exemplaire : "Que l'on m'invite à me jetter dans un gouffre comme Curtius, à tenir ma main dans un brasier comme Scaevola, à déchirer mes entrailles comme Caton, je le ferai ; mais que je m'avoue traitre à ma patrie quand j'ai bien mérité d'elle, cela n'est pas possible ${ }^{19}$. Les républicains anglais servent de même d'exempla flatteurs : " l'ombre de Sidney se présente à moi toute rayonnante de gloire... $»^{20}$. Ces références nourrissent l'éloge de Brissot par Buzot : «Sa vie fut celle d'un vrai philosophe ; il devait aussi finir comme Socrate et Sidney $»^{21}$. Émaillant son pamphlet polémique de héros républicains (Brutus, Cassius), de despotes honnis (Tibère, Caligula), de

(17) Mme Roland, op. cit., p. 396, p. 379-380.

(18) Jacques-Pierre BRISSOT, op cit. t. 1, p. 1, p. 26, p. 28, p. 41, p. 42-43.

(19) Maximin ISNARD, op. cit., p. 9.

(20) Ibidem, p. 98.

(21) Charles DaUban, op. cit, p. 15. 
philosophes (Sénèque, Socrate), d'historiens (Tite-Live, Plutarque), de figures légendaires (Prométhée, Procris), d'hommes d'État et de chefs militaires (Flamininus, Aristide...), Riouffe tâche d'augmenter la crédibilité du témoignage d'un «homme de bien». Les Mémoires de Barbaroux comportent des adieux à son enfant où la figure de Cincinnatus, retournant à sa charrue après avoir exercé la dictature dans la jeune République romaine, apparaît une promesse consolatrice :

« Ô mon fils! [...] ne venge pas ton père, venge la liberté, quelque part que tu trouves un de ces brigands qui l'ont perdue, encore dominateur, encore assassin, ou fugitif sur une terre barbare ; car quel peuple civilisé voudrait les accueillir ? Frappe, ils nous ont remis dans le droit naturel, nous, toi, les enfants de leurs malheureuses victimes. Frappe ; mais, les tyrans punis, reviens à ta charrue $[\ldots]:$ le bonheur n'est que là $»^{22}$.

Les livres que Louvet prend dans sa cache parisienne (les Géorgiques de Virgile, les Jardins de Delisle, Les Idylles de Gessner ${ }^{23}$ ) relèvent en revanche du versant bucolique des Lumières terminales, à l'aube du préromantisme.

Mme Roland confesse la révolution que fut la lecture de Rousseau :

« J'avais vingt-et-un ans, $\mathrm{j}$ 'avais beaucoup lu, je connaissais un assez grand nombre d'écrivains, historiens, littérateurs et philosophes ; mais Rousseau me fit alors une impression comparable à celle que m'avait fait Plutarque à huit ans : il sembla que c'était l'aliment qui me fût propre et l'interprète de sentiments que j'avais avant lui, mais que lui seul savait expliquer. Plutarque m'avait disposée pour devenir républicaine : il avait éveillé cette force et cette fierté qui en font le caractère ; il m'avait inspiré le véritable enthousiasme des vertus publiques et de la liberté. Rousseau me montra le bonheur domestique auquel je pouvais prétendre, et les ineffables délices que j'étais capable de goûter $»^{24}$.

Brissot se réclame de Rousseau dans sa démarche introspective dédicacée à ses enfants :

« Je touche à cet âge où Rousseau fit un examen général de sa vie passée et de toutes ses connaissances, et se fixa un plan de conduite qu'il pût suivre jusqu'à sa mort. Je vais imiter Rousseau ; j'en ai le loisir, et je

(22) Charles BARBAROUX, op. cit., p. 82.

(23) Jean-Baptiste LOUVET, op. cit., p.180.

(24) Mme ROLAND, op. cit., p.464. 
remplirai par là plusieurs objets : premièrement de m'améliorer et de me fixer pour le reste de ma vie, si le ciel m'accorde encore quelques années ; secondement, d'occuper agréablement et utilement des heures qu'il est absurde de laisser dévorer par le chagrin et par l'ennui ; troisièmement, d'être utile à mes enfants, car c'est principalement pour eux que j'écris. Je veux leur apprendre à connaître leur père, je veux les dédommager des instructions que je n'ai pas pu leur donner lorsque j'étais encore entraîné par le tourbillon des affaires. Vous quitter si tôt! Vous que j'ai à peine entrevus, vous que mes occupations ont empêché de soigner, d'élever moi-même ! Vous quitter au moment où, rompant mes liaisons politiques, j'allais me consacrer à votre éducation, et mériter votre tendresse en me montrant votre père ! »

Il mime en effet Rousseau sur les plaisirs familiaux par exemple : «Ces bois solitaires favorisaient la méditation dont j'étais avide. Là, ne craignant que les mosquites [sic] et à l'abri des importuns et des importants de la ville, je me livrais avec mon épouse au doux plaisir d'être le témoin des ébats de notre enfant. Là, mon cœur palpitait à l'aspect de la nature. Je n'ai jamais vu les bois, une solitude épaisse, sans ressentir un tressaillement, une satisfaction intérieure et le désir de ne m'en jamais séparer. Je n'entrevoyais qu'avec horreur le moment de la quitter pour retourner à la ville ». Entre Rousseau et Voltaire, qu'on dit plus proche des girondins, Brissot ne balance pas :

« Comme le caractère de l'Aristippe moderne me paraît à nu dans ses mémoires ! On l'y voit louer, admirer en public un prince dont il ravale en secret le mérite, dont il ridiculise les vices ; on le voit jeter l'opprobre et le ridicule à pleines mains sur une foule de personnages qui en versent encore aujourd'hui des larmes ; on le voit détruire par ses satires les réputations qu'il avait créées par ses éloges ; barbouiller de fumée les idoles qu'il avait parfumées de son encens ; on le voit ironique, méchant, et s'applaudissant de ses méchancetés et de ses sarcasmes. Comparez le donc à Jean-Jacques ! Celui-ci est faible, et il s'accuse ; celui-là est vicieux et coupable, et il s'élève et se pavane. Certes, nul que moi n'admire plus le génie de Voltaire, et ne lui tient plus de compte du bien qu'il a fait à la philosophie et à l'humanité ; dans plus d'un de mes écrits, j'ai prouvé cette admiration ; mais entre son génie et son cœur, entre ses confessions et celles de Rousseau, je crois qu'il y a un intervalle $»^{25}$.

(25) Jacques-Pierre BRISSOT, op. cit., t. 1, p. 24, p. 251-252 ; t. 2, p. 44. 
Isnard qui cite le «Discours sur le Pacte Social » [sic], affecte une misanthropie tempérée par la joie des trouvailles familiales : «depuis mon retour parmi les hommes, chaque fois que j'éprouve un peu trop les tracasseries qui naissent du frottement social, je me dis en secret : Ah! tu n'éprouvais pas tout cela dans ton souterrain... ! Mais je vais bientôt embrasser un père, une mère, une femme, des enfants, qui, depuis 15 mois, pleuraient ma mort; et voilà ce qui va rendre ma vie actuelle une source de délices $»^{26}$.

Comme Meillan, proche de l'Espagne, et Isnard de l'Italie, aux pudeurs suspectes sur les caches en l'an II, Louvet déguise peut-être son émigration en Suisse ${ }^{27}$ sous un Jura de fiction où il songe à Rousseau :

«Afin de donner le change à mes vives agitations, je foule de mes pieds impatients cette terre agreste, avec rapidité je parcours les silencieux labyrinthes de ces retraites ; avec effort je gravis les énormes roches jetées sans ordre, taillées à pic, chargées de chênes immenses; bientôt comme suspendu sur les bords les plus élevés de cet abîme, au fond duquel un torrent innavigable roule à grand bruit son onde antédiluvienne, je me retrouve, je pense, je donne de l'essor à mes idées les plus hardies : quel mortel viendrait ici jusqu'à moi ? Ici, loin des hommes et devant Dieu, malgré toutes les révolutions, en dépit de tous les tyrans, je suis encore moi, je suis libre! Mais ô tourment ! quelques hommes se montrent [...] il me faut soudain quitter ces hauteurs, m'enfoncer dans le plus épais du bois, retrouver mon dernier asile ; ou malheur à moi !... Alors, je me rappelle que ce fut ton sort, ô mon maître, ô mon soutien, sublime et vertueux Rousseau. Toi aussi, pour avoir bien mérité du genre humain, tu t'en vis persécuté. [...] Tu m'as donné l'exemple de porter encore le poids de la vie $»^{28}$.

Il lance cet avertissement qui ne vaut que pour la prégnance acquise par le modèle rousseauiste du pacte de lecture entre un auteur feignant de dire la vérité et son lecteur feignant de le croire : «Qu'on s'attende à la vérité, car je proteste que, pénétré de respect pour elle, je regarderais comme un crime la seule pensée de l'altérer $»^{29}$. Meillan respecte aussi cette loi du genre - «Je proteste néanmoins que je ne dirai rien qui ne soit rigoureusement vrai $»^{30}$, de même que Riouffe, avec des accents

(26) Maximin ISNARD, op. cit., p. 10, 20-21.

(27) D'après Auguste DANICAN, Les brigands démasqués, ou Mémoire pour servir à l'histoire du temps présent, Londres, Deboffe, 1796, p. 72.

(28) Jean-Batiste LOUVET, op. cit., p.195-196

(29) Ibidem, p. 11.

(30) Arnaud-Jean MEILlan, op. cit., p. 57. 
thermidoriens : « Je peins les objets tels que je les ai vus. [...] La vérité ne connaît point d'amnistie $»^{31}$.

Brissot déplore l'influence sur sa famille de «l'infernal esprit du sacerdoce : il sème la haine sur le sol de l'amitié ; il substitue des poignards aux fleurs dont elle s'entoure ». Par l'évocation de «cet autre monde, auquel je crois, et que je ne définis pas », il montre un déisme, compatible avec l'anticléricalisme, mais ne va pas jusqu'à l'athéisme, soutenant que ses interrogations religieuses se résolurent à la lecture du Vicaire savoyard:

« Je me couchais matérialiste et je me réveillais déiste ; le lendemain je donnai la pomme au pyrrhonisme. Quand j'avais la fierté de l'esprit fort, l'athéisme me plaisait davantage. Plus je m'éloignais des prêtres, plus je me croyais prêt de la vérité. Lorsque la voie intérieure se faisait entendre, lorsque je l'écoutais, alors j'étais convaincu de l'existence de l'Être suprême, je lui adressai des prières avec ferveur. Mais si j'avais recours au raisonnement, alors tout me devenait incertitude [...] Tel était l'état de doute et d'erreur où j'ai passé quelques-unes de mes années, jusqu'enfin éclairé par les ouvrages de Jean-Jacques Rousseau, ayant mûrement réfléchi le témoignage de mon sens intime, j'ai pris le parti de croire à un Dieu, et de régler ma conduite en conséquence $»^{32}$.

Louvet ponctue l'évocation des épreuves ou du bonheur retrouvé d'invocations religieuses louant une transcendance créatrice et bienfaisante dont il lit la preuve de l'existence dans l'amour retrouvé : « Dieu protecteur, grâces te soient rendues ; elle est de retour. C'est sous ses yeux que je jette ces dernières lignes. Il est donc certain qu'il existe une Providence rémunératrice $»^{33}$. Isnard assure « qu'il est une providence consolatrice de la vertu outragée » et livre ces émois métaphysiques peu voltairiens :

« Je me promenai seul dans un jardin environ trois heures chaque nuit ; le spectacle de la voûte étoilée, le seul qui s'offrit à ma vue, fixoit presque continuellement mes réflexions. Ah, quelles étaient salutaires et ravissantes...! Qu'il est sublime ce livre sans cesse ouvert sur nos têtes, tracé de la propre main de l'être incréé, et dont chaque lettre est un astre ! Qu'il est heureux celui qui sait lire ce que j'y voyois écrit en traits de

(31) Honoré RIOUFFE, op. cit., p. 2

(32) Jacques-Pierre BRISSOT, op. cit., p. 28-29, 30, p. 38-39, p. 60.

(33) Jean-Baptiste LOUVET, op. cit., p. 72, p. 201. 
feu, en hiéroglyphes solitaires : Existence de Dieu. Immortalité de l'âme. Nécessité de la vertu $»^{34}$.

Mme Roland présente un esprit très libre qui considère « la source des vertus humaines, fort indépendante de tout système religieux, des billevesées de la métaphysique, et des impostures des prêtres » :

«La belle idée d'un Dieu créateur dont la Providence veille sur le monde, la spiritualité de l'âme, son immortalité, cet espoir consolateur de la vertu persécutée, ne seraient-elles que d'aimables chimères ? Que de nuages environnent ces questions difficiles ! [...] Mais qu'importe à l'âme sensible de ne pouvoir les démontrer? Ne lui suffit-il pas de les sentir! Dans le silence du cabinet et la sécheresse de la discussion, je conviendrai avec l'athée ou le matérialiste de l'insolubilité de certaines questions ; mais au milieu de la campagne et dans la contemplation de la nature, mon cœur ému s'élève au principe vivifiant qui les anime, à l'intelligence qui les ordonne, à la bonté qui m'y fait trouver tant de charmes $»^{35}$.

Buzot pense peut-être à elle dans cette perspective hypothétique : «si, comme l'ont pensé les sages, il existe après cette vie un lieu où les amis de la vertu sont récompensés des maux qu'ils ont souffert pour elle...» ${ }^{36}$. Riouffe qui fanfaronne avec la fausse religion d' «Ibrasha », évoque pourtant une puissance tutélaire : «Je ne sais quelle providence qui a toujours mesuré mes adversités à mes forces, m'a épargné cet horrible malheur, le plus grand qui puisse arriver, celui de causer la perte de ses amis $! »^{37}$.

Dans leur goût des paysages, les girondins manifestent une sensibilité qui ne relève plus tout à fait des Lumières. Buzot brosse un tableau déjà romantique de sa vie de proscrit :

« Dans ma vie errante, incertaine, solitaire, parcourant du nord au midi, les montagnes, les mers, les lieux les moins fréquentés d'hommes, exposés aux intempéries de l'air, à la rigueur des saisons, souvent sans pain, sans aucune nourriture, sans vêtement et sans argent ...» ${ }^{38}$.

(34) Maximin ISNARD, op. cit., p. 21, p. 22-23.

(35) Mme RolAnd, op. cit., p. 397-398.

(36) Charles DAUBAN, op. cit., p. 3.

(37) Honoré RIOUFFE, op. cit., p. 139.

(38) Charles DaUBAn, op. cit., p. 34. 
Louvet décrit des paysages montagneux changeant comme ses états d'âme. Dans l'attente angoissée de sa maîtresse, il évoque un vertige qui n'est pas sans rappeler l'évocation du Pas de l'Échelle dans les Confessions :

«Avec l'aurore, j'allais me jeter dans ces bois naguère seulement mélancoliques, maintenant tristes, sombres, plein d'horreurs. Sur ces roches où dernièrement je me bornais à fuir les hommes, aujourd'hui je venais chercher les images du Chaos et de la destruction. Que de fois j'ai, d'un œil d'envie, mesuré ces deux cents pieds d'élévation, d'où je pouvais, me précipitant, rouler de pierres en pierres, et déjà mille fois brisé, m'engouffrer dans ces eaux rapides, tempétueuses, blanchies d'écume et d'ailleurs trop peu profondes pour empêcher que de tout mon poids centuplé par la chute, je n'achevasse de me mettre en pièces sur le tranchant du roc vif qui formait leur lit !».

Avec Lodoïska en revanche, les forêts alpines deviennent chambre nuptiale, origine du monde, promesse de félicité :

« J'ai découvert entre ce bois touffu qui [...] se présente en amphithéâtre, par mille détours monte peu à peu vers la plaine, la couvre tout entière, et d'une pente insensible se prolonge jusqu'à la vallée ; entre ces roches qui [....] élèvent, taillé par ainsi dire à pic, leur inabordable rempart chargé de forêts éternelles ; près de ces eaux qui plus loin resserrées se précipitent impétueux torrent, mais ici libres dans un vaste espace s'écoulent ruisseau paisible [...], j'ai découvert l'asyle enchanteur. [...] Je t'y mènerai dans ce bosquet, Lodoïska ; à travers les nombreux détours du labyrinthe qui le masque, je serai ton guide. [...] Je viens d'écarter quelques branches, regarde : voilà cette difficile entrée ; tu n'aperçois rien encore ? Avance, approche-toi, baisse-toi. Passe inclinée sous ces pesants rameaux que je soulève, sous cet arc triomphal que mon bras soulève pour toi $»^{39}$.

\section{Des Mémoires de combat}

Par delà cette légèreté qui n'appartient qu'à la plume de Faublas retrouvée en l'an III dans une culture moins différente de celle des montagnards qu'on s'est plu à l'écrire, les girondins, font aussi de leurs Mémoires des textes de défense et d'attaque.

La violence verbale ne constituant pas l'apanage des adversaires qu'ils entendent disqualifier, ils dépeignent de sombres galeries des affreux.

(39) Jean-Baptiste LOUVET, op. cit., p. 200. 
Ainsi Manon Roland anathématise-t-elle : «O Danton ! [...] tu aiguises les couteaux contre tes victimes. Frappe ! une de plus augmentera peu tes crimes ; mais leur multiplicité ne peut couvrir leur scélératesse, ni te sauver de l'infamie. Aussi cruel que Marius, plus affreux que Catilina, tu surpasses leurs forfaits sans avoir leurs grandes qualités, et l'histoire vomira ton nom avec horreur dans le récit des boucheries de Septembre et la dissolution du corps social à la suite des événements du 2 juin ». Elle en réserve autant à Fabre d'Églantine : «affublé d'un froc, armé d'un stylet, occupé d'une trame pour décrier l'innocence ou perdre le riche, dont il convoite la fortune, il est si parfaitement dans son rôle que quiconque voudrait peindre le plus scélérat Tartuffe n'aurait qu'à faire son portrait ainsi costumé ». Elle conspue l'apparence et le caractère des militants populaires, ces « grands sans-culottes à cheveux puants », « un essaim d'hommes peu connus, intrigants de sections ou braillards de clubs, patriotes par exaltation et plus encore par intérêt $»^{40}$.

Buzot n'a pas de mots assez durs pour les « assemblées sectionnaires de Paris composées de quelques individus imbéciles ou scélérats, qui s'étaient emparés audacieusement de la volonté de tous les Parisiens » et « le gouffre pestilentiel des Jacobins, comme un antre du méphitisme ou de la corruption ». Il dénonce le détournement du décret punissant de mort quiconque proposerait le rétablissement de la royauté : «Les Jacobins s'emparèrent du principe comme les corbeaux d'une charogne puante, et rien ne fut capable de les chasser de cette horrible pâture ». Les traits rageurs de Buzot montrent que la stigmatisation physiognomonique et politique des montagnards ne commence pas en Thermidor :

« Marat que la nature semblait avoir formé pour rassembler en un seul individu tous les vices de l'espèce, laid comme le crime, qu'il suait par tous les pores de son corps hideux et pourri par la débauche, bête féroce, poltron et sanguinaire, ne parlait que de sang, ne prêchait que l'effusion du sang, ne se délectait que dans le sang. Ce monstre qui a fait secte en France au dix-huitième siècle, n'avait au lieu de talents que de l'impudence et de la férocité. Son apothéose sera un jour la satire la plus amère de la révolution de 1793, et son nom, en exécration à la postérité, couvrira d'opprobre la nation qui le déifia $»^{41}$.

(40) Mme Roland, op. cit., p. 113, p. 271, p. 77, p. 119.

(41) Harles DAUBAN, op. cit., p. 30, p. 69. 
Les mots de Louvet constituent un modèle de la virulence thermidorienne :

« Du milieu de la Montagne jusqu'à son sommet, c'étaient l'ignorance présomptueuse prétendant à tous les profits de la célébrité, l'avide cupidité aspirant aux richesses, la crapule vile espérant de longues débauches, la vengeance atroce préparant des assassinats, la basse vie désespérée de l'influence du talent, l'insatiable ambition dévorée du besoin de régner au prix de tous les forfaits. Et lorsque de tels scélérats commencèrent à l'emporter, lorsque sur des monceaux de dépouilles, sur les débris de toutes propriétés, la foule à leur voix obéissante se baigna dans des flots de sang innocent, lorsque le pillage organisé par les magistrats, l'athéisme réduit en principe, et deux cents mille échafauds ordonnés par les lois, souillèrent ma patrie, je fus obligé de reconnaître que, de toutes les espèces de servitude, celle que l'anarchie produit est encore la plus intolérable ».

Fantasmant sur la possibilité du retour des «hommes de sang », Louvet va, sans crainte du paradoxe, jusqu'à invoquer l'élimination physique de ceux auxquels il reproche la Terreur : « que cette race de mangeurs d'hommes soit exterminée ! $\gg^{42}$.

Les girondins plaident leur défense en valorisant leur dévouement à la Révolution, comme Barbaroux qui décrit son apothéose après le 10 Août : « Tous les citoyens se mirent à genoux dans la maison et dans la rue. J'étais alors debout sur une chaise où l'on me retint : Dieu ! quel spectacle ! des larmes coulèrent de mes yeux. Si je fus pour eux en ce moment comme la statue de la liberté, je puis m'honorer au moins de l'avoir défendue de tout mon courage $\gg^{43}$. Buzot souligne l'intransigeance de son civisme : « tous mes discours, toutes mes démarches, tous mes vœux ont été pour l'union de la République comme pour l'égalité entre les départements, la liberté de tous les citoyens, le bonheur et la gloire de mon pays $»^{44}$. Louvet romance son enthousiasme politique :

« Cette révolution [...] elle était belle et juste. Le moyen de ne pas nous passionner pour elle, au détriment même de notre intérêt le plus cher ? J'en serai quitte, disais-je à mon amie, pour faire quelque autre ouvrage et travailler un peu plus de temps. Si le retardement apporté à notre bonheur

(42) Jean-Baptiste LOUVET, op. cit., p. 69-70 ; p. 13-14.

(43) Charles BARBAROUX, op. cit., p. 85.

(44) Charles DAUBAN, op. cit., p. 58. 
produit le bonheur du genre humain, pourrions-nous ne pas trouver quelque douceur dans nos sacrifices ? $\gg^{45}$

Mme Roland gémit des espérances déçues : « Sublimes illusions, sacrifices généreux, bonheur, patrie, adieu! Dans les premiers élans de mon jeune cœur, je pleurais à douze ans de ne pas être née Spartiate ou Romaine ; j'ai cru voir dans la Révolution française l'application inespérée des principes dont je m'étais nourrie $\gg^{46}$.

Leurs Mémoires visent à camper les girondins en héros républicains, mais aussi à se justifier du fédéralisme que Manon Roland considère « un épouvantail pour les sots $»^{47}$. Brissot dédouane les girondins de l'accusation d'hostilité envers Paris, en dénonçant l'instrumentalisation du peuple de la capitale :

«Si nous nous sommes élevés avec tant de force et tant de constance contre les massacres de septembre, [...] ce n'était pas pour flétrir les auteurs de la Révolution du 10 août, ni les Marseillais, ni les Brestois, ni les Parisiens qui ont renversé la tyrannie et n'ont pas souillé leurs mains dans le sang des prisonniers ; ce n'était point pour déshonorer cette révolution du 10 Août, mais pour la venger ; et on ne le pouvait qu'en séparant les massacres de septembre de l'immortelle révolution du 10 août ; ce n'était point pour calomnier le peuple de Paris, mais bien au contraire pour le justifier, lui que l'on avait accusé d'être le complice de ces exécrables attentats ; c'était pour laver le gouvernement républicain de cette tâche que lui prêtaient les puissances étrangères afin d'indigner contre nous leurs peuples, afin de rendre à jamais exécrable la Révolution française $»^{48}$.

Buzot qui n'est pas le dernier à invectiver, s'indigne des techniques de disqualification des montagnards : " Nos adversaires ont bien su qu'avec des mots on répondait à tout. [...] Il ne s'agit pas de savoir si la chose est vraie ; il est utile de le dire, et cela suffit ; à force de le répéter, il faudra bien qu'on le croie. Ce sont là les règles de conduite de nos adversaires : elles leur ont bien réussi ; il n'est pas un département, pas une ville, pas un misérable club qui ne nous traite de royalistes et de fédéralistes ». Il illustre sa dénonciation d'une fable :

(45) Jean-Baptiste LOUVET, op. cit., p. 16.

(46) Mme ROLAND, op. cit., p. 74-75.

(47) Ibidem, p. 126.

(48) Jacques-Pierre BRISSOT, op. cit., p. 244. 
«Un jour, je fus accueilli très hospitalièrement chez un bonhomme de ce pays qui passait pour un des coryphées de la société populaire. Nous causions tranquillement au coin du feu sur les affaires du temps. Citoyen, me dit-il, je hais les fédéralistes, mais je n'aime pas non plus qu'on tue comme ça des hommes sans les entendre. Je lui dis que nous n'avions pas donné lieu qu'on nous imputât le projet d'établir le fédéralisme en France [...]. Il imaginait je ne sais quoi de monstrueux, de terrible, de contre-révolutionnaire, sous ce nom de fédéralisme, qu'il haïssait à l'égal de la misère et de la faim $»^{49}$.

Dans une philippique sentant le barreau, rythmée par la répétition de l'interrogation «Fédéralistes ! Et pourquoi ? », il entend démontrer comment les mesures proposées par les girondins - garde départementale pour l'Assemblée, appel au peuple dans le procès du roi, enquête sur les massacres de Septembre...- ont été dévoyées pour étayer un crime imaginaire. Passé Thermidor, Riouffe reprend ce raisonnement : «Le prétendu fédéralisme fut un vaste piège, dans lequel furent enveloppés tous les administrateurs dignes de leur poste, et une foule d'hommes dignes de la liberté $»^{50}$. De même Louvet défend-il la thèse de l'invention diffamatoire :

«Certes, il exista le fédéralisme, [...] mais il n'exista que par vous, tyrans, et pour vous. Cependant, s'écrièrent quelques hommes abusés, les départements se sont fédéralisés pour marcher contre la Convention. Contre la Convention ! Jamais ! Pour elle, toujours. Mais d'ailleurs fédéralisés ? Que voulez-vous dire ? Elles étaient donc fédéralistes, au 14 juillet, les sections de Paris qui chacune en particulier trop faible, se fédérèrent pour renverser la Bastille ? Ils étaient donc fédéralistes, le 10 août, ce bataillon du Finistère, ce bataillon de Marseille et ces nombreux bataillons de Paris qui se fédérèrent contre le château ? [...] Enfin, se fédérer, c'est donc se fédéraliser ? Quel misérable abus de mots ! ${ }^{51}$

Ces mémorialistes prétendent faire la vérité sur les projets de leurs adversaires et inaugurent dès 1793 la théorie, généralisée dans la réaction thermidorienne et la dépolitisation du Directoire ${ }^{52}$, de la contre-révolution

(49) Charles Dauban, op. cit., p. 52.

(50) Honoré RIOUFFE, op.cit., p. 2.

(51) Jean-Baptiste LOUVET, op. cit., p. 29-30.

(52) Marc DelEPlace, L'anarchie de Mably à Proudhon (1750-1850). Histoire d'une appropriation polémique, Lyon, ENS Éditions, 2000 ; Pierre SERNA, La République des girouettes. 1795-1815 et au-delà : une anomalie française : la république de l'extrême centre, Paris, Champ Vallon, 2005 ; Jean-Luc CHAPPEY, « Révolution, régénérations, civilisation : enjeux culturels des dynamiques 
par l'anarchie. Ainsi Mme Roland apostrophe-t-elle «messeigneurs les Jacobins, devenus fous, enragés ou stipendiés par les ennemis $»^{53}$. Buzot qui estime que « on sait à présent ce qu'on peut faire des Français avec la terreur, et le secret ne sera pas perdu pour les rois », se targue de faire tomber le masque : "Voilà les hommes qui, avec le fourbe et lâche Barère, font la destinée de la France ! Si nous eussions été des contre-révolutionnaires, il eût fallu se les associer » ${ }^{54}$. Louvet reprend cette allégation : " l'Autriche et l'Angleterre avaient leurs principaux agents dans les Jacobins ». Il répète la rumeur du rétablissement de la royauté imputée à Robespierre en évoquant « ce trône où maintenant il touche de sa main hypocrite, calomniatrice et sanglante ; de ce trône où il ne lui faut plus qu'un forfait pour s'asseoir $»^{55}$. Meillan relaie l'invention par les thermidoriens, « libérateurs de la patrie », d'un « système de terreur » pratiqué par « des reptiles qui s'agitent en vain dans la fange du crime $»^{56}$, de même que Riouffe qui requiert - «Pour nous Robespierre fut toujours un tyran, le 31 Mai une contre-révolution, la montagne un ramas de brigans ou de fous furieux, les jurés du tribunal révolutionnaire des cannibales $»^{57}$. Emboîtant le pas de Barère, Lecointre et Tallien $^{58}$, il relaie l'invention d'un « système de Terreur » en essentialisant Prairial : «Les horreurs dont ils ont épouvanté le monde, n'étaient que le prélude d'un plan infernal, auquel ils paraissent s'être arrêtés définitivement un mois ou deux avant le 9 Thermidor $»^{59}$.

Ces mémorialistes affirment enfin que le peuple a été instrumentalisé, comme Brissot qui lance ces questions de prétoire : «Était-ce calomnier Paris, que de dire à satiété que l'immense majorité en était bonne, qu'elle voulait la loi, la soumission à la Convention ; que de distinguer l'immense majorité de ses habitants de la poignée de brigands qui infestaient ses murs, qui troublaient la tranquillité, excitaient le tumulte, insultaient à la Convention ? $»^{60}$. Renonçant comme Riouffe à soigner le peuple - « Le jacobinisme et le robespierrisme étaient les maladies nouvelles dont on

politiques », Pourquoi faire la Révolution ? Jean-Luc CHAPPEY, Bernard GAINOT, Guillaume MAZEAU et al, Marseille, Agone, 2012, p. 115-148.

(53) Mme RolAND, op.cit., p. 49

(54) Charles DAUBAN, op. cit., p. 6, P 69-70.

(55) Jean-Baptiste LOUVET, op. cit.,, p. 33-34, p. 45.

(56) Arnaud-Jean MeILlan, op. cit., p. 29, p. 109-110.

(57) Honoré RIOUFFE, op. cit., p.150.

(58) Françoise BRUNEL, «Bridging the Gulf of the Terror » dans Keith Michael BAKER (dir.), The French Revolution and the Creation of Modern Political Culture, vol. 4, The Terror, Oxford, Pergamon Press, 1994, p. 327-346 ; Sophie WAHNICH ; «La question de la responsabilité collective en l'an III dans Michel Vovelle (dir.), Le tournant de l'an III. Réaction et Terreur blanche dans la France révolutionnaire, Paris, CTHS, p. 85-97. Pierre SERNA, op. cit.

(59) Honoré RIOUFFE, op. cit. p. VII.

(60) Jacques-Pierre BRISSOT, op. cit., p. 242-243. 
voyait bien les symptômes, mais dont on ignorait les terribles effets $»^{61}-$ les girondins en conçoivent une vive amertume, tel Barbaroux qui livre ces aigres assertions :

« Le peuple ne mérite pas qu'on s'attache à lui, car il est essentiellement ingrat ; ni qu'on défende ses droits, car on en abuse. J'ai vu les Français, sensibles hier, boire le sang des plus hommes de bien. Il faut servir nos semblables par l'exemple de nos vertus ; s'ils souffrent, il faut les secourir : mais vouloir conduire à la liberté un peuple sans mœurs, qui blasphème Dieu et adore Marat, c'est la plus absurde folie. Cette populace n'est pas plus faite pour un gouvernement philosophique, que les Lazzaroni de Naples et les anthropophages de l'Amérique $»^{62}$.

Brissot se désole, voyant à Montargis des « êtres à figures hideuses qui laissaient entrevoir un regret, celui de ne pas boire mon sang à l'instant même. Rien n'a mieux peint le spectacle d'une danse de cannibales autour de leur victime attachée au fatal poteau, que la vue de ces monstres qui m'annonçaient avec un air de jubilation la guillotine prochaine... je ne fus pas inquiet malgré leurs fureurs, mais mon âme était navrée. - Voilà donc, me disais-je, les hommes pour lesquels tu as sacrifié tes veilles, ton repos, ta famille, tout ce que tu as de plus cher ! Fasse le ciel, m'écriai-je, la larme à l'œil, que mon exemple ne décourage pas les vrais amis de la liberté ! $»^{63}$. Buzot dit désespérer du peuple : «En vérité, c'est folie, j'en ai du moins grand peur, de vouloir servir le peuple par des moyens honnêtes ; la vérité n'est pas faite pour lui. Il ne lui faut que vent et fumée, c'est là sa pâture ». Il en conçoit une radicale exécration de Paris : «La France républicaine avec une capitale, la France libre avec Paris, siège principal de ses établissements nationaux, une république française avec une législation et un gouvernement à Paris ? En vérité, il faut être en délire pour concevoir de pareilles absurdités ! [...] La France ne peut espérer ni liberté ni bonheur que dans la destruction entière et irréparable de cette capitale ${ }^{64}$.

\section{Corps et âmes dans la Révolution}

Défense et illustration des girondins, leurs Mémoires présentent les liens douloureux de ces individus à l'histoire dans laquelle ils espèrent vivre

(61) Charles BarbarouX, op. cit., p. 7.

(62) Charles BARABOUX, op.cit., p. 82.

(63) Jacques-Pierre BRISSOT, op. cit., p. 228.

(64) Charles DAUBAN, op. cit., p. 50, p. 62, p. 24. 
encore. Les causes de leur échec sont peu analysées par Brissot : « Hélas ! Il était si facile d'arriver à la concorde ! Si facile d'établir enfin la liberté et de la rendre durable, en ne se fondant que sur la raison, sur l'évidence et sur l'ordre! Par quelle fatalité la philosophie, qui avait renversé le despotisme, était-elle à son tour foulée aux pieds par le vandalisme et le cannibalisme ? ${ }^{65}$. Buzot cède à l'illusion de la nécessité : «il était aisé de prévoir ce qui est arrivé ; [...] avec un tel peuple et dans de telles circonstances, il fallait que l'audace du crime l'emportât à la longue ; et la France, après avoir passé par toutes les horreurs de la licence et de l'anarchie, devait enfin périr ». Dédouanant les girondins, il considère qu'une meilleure information aurait prévenu les événements qu'il déplore :

«Les premiers torts sont aux journalistes du temps, aux députés surtout de l'Assemblée législative, qui n'instruisirent leurs départements ni de la position où se trouvait l'Assemblée, ni de l'état de la ville de Paris. [...] S'ils eussent fait parvenir la vérité jusqu'à nous, s'ils eussent osé la dire aux assemblées électorales, la France était sauvée, avec la liberté, la paix, le bonheur. Une seule résolution prise dans les assemblées électorales eût suffi pour opérer cela. Il ne fallait qu'enjoindre aux députés de se réunir ailleurs que dans Paris, et Marat, Danton, Robespierre et leurs associés n'entraient point dans le sein de la Convention nationale ; on ne prévit rien, la Convention vint à Paris, et dès lors tout fut perdu $»^{66}$.

Pétion imagine une autre solution : « les départements [...] pouvaient employer une autre force qui n'était pas moins puissante, c'était celle de l'inertie; s'ils avaient refusé l'exécution des décrets, l'empire des scélérats finissait, et il fallait nommer une Convention nouvelle ; la punition des traîtres était une suite inévitable de cette marche ${ }^{67}$. Meillan n'est pas plus réaliste :

«Quelque faible que fût encore la force départementale, si elle avait pu se rendre à Paris avant que la Montagne n'eût eu le temps de revenir de sa première terreur, elle eût offert aux Parisiens un point d'appui qui leur manquait et qu'ils réclamaient ouvertement. Il n'en fallait pas davantage. Il suffisait d'arriver et tout rentrait dans l'ordre. Alors point de guerre civile,

(65) Jacques-Pierre BRISSOT, op. cit., t. 2, p. 219.

(66) Charles DAUBAN, op. cit., p. 3-4, P. 66-67.

(67) Ibidem, p. 152. 
point de fédéralisme. Les accusations retombaient toutes sur la Montagne et nous étions comblés de bénédictions ${ }^{68}$.

Louvet qui réfléchit après coup, se montre plus perspicace : « À Marseille, à Bordeaux, dans presque toutes les villes principales, le propriétaire lent, insouciant, timide, ne pouvait se résoudre à quitter un instant ses foyers ; c'étaient des mercenaires qu'il chargeait de sa querelle et de ses armes ». Mais il s'imagine avec complaisance en premier rôle, à propos notamment de son discours contre Robespierre le 29 octobre 1792 :

« L'accusation que j'intentai produisit le plus grand effet. [...] Le lâche crut sa dernière heure arrivée, il vint à la tribune me demander grâce. [...] Si Pétion que j'interpellai plusieurs fois, eût voulu dire le quart de ce qu'il savait, Robespierre et son complice étaient décrétés sur l'heure. Alors, détestés dans la République entière, n'ayant dans Paris qu'un parti très inférieur à celui de la Convention, ils recevaient le châtiment de leurs crimes. L'infâme Orléans et une vingtaine de brigands subalternes rentraient dans leur nullité ; un Barère, un Delacroix, un ramas de vils intrigants toujours prêts à traîner le char du parti dominant, restaient Rolandistes ; la République était sauvée ${ }^{69}$.

Certains girondins entretiennent d'excellents rapports à soi, tel Isnard qui n'a pas un mot pour ses défunts collègues mais emprunte les mots de Brissot pour Danton, se décrivant ainsi dans son hagiographie : «Moi, ce courageux athlète de la liberté, ce vétéran tout couvert de cicatrices de la révolution, qu'on immolerait aujourd'hui, parce que jadis il parla avec force aux conspirateurs du 9 thermidor ? Quoi ! Ceux qui les ont combattu les derniers sont des héros, et moi je serais criminel ?... » ${ }^{70}$ Buzot se méfie au contraire des mirages autobiographiques : « Ce travail est extrêmement délicat, il exige beaucoup de soin et il n'est pas donné à tout le monde d'inspirer un intérêt soutenu en parlant trop de soi ; j'ai voulu le mériter avant d'y prétendre. Je craindrais aussi d'être trop indulgent envers moi-même, et quoique je sois, je ne veux pas qu'on me prenne pour un autre $»^{71}$.

Leurs Mémoires évoquent les expériences vécues en l'an II. Louvet valorise sa bonne nature dans la retraite vers la Bretagne : « Je trouvais,

(68) Arnaud Jean MeILlan, op. cit., p. 79.

(69) Jean-Baptiste LOUVET, op. cit., p. 72, p. 32.

(70) Maximin ISNARD, op. cit., 92-93

(71) Charles DaubAn, op. cit., p. 3. 
pour moi, fort agréable de faire avec ces braves gens ma journée à pied, de boire et manger avec eux, sur la route, le verre de cidre, le petit morceau de beurre et le pain de munition ; puis, à la couchée, d'aller avec un billet, prendre modestement mon logement chez un particulier, qui, me croyant un volontaire, ne se gênait nullement avec moi, et me dispensait par là de toute espèce de cérémonie ». Mais il souligne les faiblesses physiques de ses collègues :

« Cussy, tourmenté d'un accès de goutte, gémissait à chaque pas qu'il fallait faire ; Buzot, débarrassé de ses armes, était encore trop pesant ; non moins lourd, mais toujours plus courageux, Barbaroux, à vingt-huit ans gros et gras comme un homme de quarante, et pour comble de mal ayant attrapé une entorse, se traînait avec effort, appuyé tantôt sur mon bras, tantôt sur celui de Pétion et Salles, également infatigables ; enfin Riouffe ayant été forcé de quitter ses bottes trop étroites qui l'avaient blessé, se voyait obligé de sauter sur la pointe de ses pieds nus, dont les talons étaient écorchés ».

Il brode sur les joies d'un asile offert par un curé en Gironde : « Le repas ici n'était rien, auprès des touchantes attentions qui les précédèrent : c'était de l'eau tiède pour laver nos pieds, un grand feu pour nous sécher, tout l'attirail d'une toilette pour couper nos longues barbes et rafraichir nos chevelures, du linge blanc pour nous changer, enfin des viandes légères et du vin restaurant que vous versait une jolie nièce ! [...] Nous trouvions notre sort semblable à celui de ces fiers paladins qui venant de combattre des monstres, rencontrent tout à coup, dans quelque pavillon enchanté, des fées pour les servir ». C'est pour mieux enténébrer le puits de Mme Bouquey à Saint-Émilion «à trente pieds sous terre, et l'entrée [du] souterrain, d'ailleurs fort dangereuse, était encore si bien masquée qu'on ne la pouvait découvrir. Quelque spacieux que fût le caveau, le séjour continuel de cinq hommes pouvait y corrompre l'air qui ne se renouvelait que très difficilement. Nous nous pratiquâmes, dans une autre partie de la maison, une seconde forteresse, plus saine, presqu'aussi sûre, presque aussi difficile à découvrir ». Chez elle, il peint une cène girondine : « Pour ne pas déjeuner, on ne se levait qu'à midi. Une soupe aux légumes faisait tout le dîner. [...] Tantôt un morceau de bœuf obtenu à grand peine à la boucherie, tantôt une pièce de la basse-cour bientôt épuisée, quelques œufs, quelques légumes, un peu de lait composaient le souper dont elle ne s'obstinait à prendre qu'un peu, pour nous en laisser davantage. Elle était au milieu de nous comme une mère environnée de ses enfants pour lesquels elle se sacrifie ». 
Des parenthèses enchantées ponctuent les romanesques pérégrinations de Louvet qui célèbre le bonheur quotidien de l'amour à Paris :

«Qu'il sera délicieux, ce repas qu'elle apprête de ses mains charmantes! Au moins, c'est moi qui mets le couvert! C'est moi qui dois servir à table, quoique je le fasse bien maladroitement, car je n'y vois goutte. Mais j'ai mes raisons pour m'y obstiner : de peur qu'il ne m'en reste point assez, elle me donnera tout si je la laisse faire, et si quelquefois je ne me fâche. Après dîner, c'est elle qui me fait tout haut la lecture, puis elle est à son piano ; ensuite une partie d'échecs ; et parmi tout cela de doux entretiens à voix basse. Enfin nous soupons encore tête à tête, car peu de gens sont curieux de troubler notre périlleuse retraite ; et nous nous couchons, souhaitant avec ardeur que des barbares ne viennent pas nous ravir la superbe journée du lendemain $»^{72}$.

Riouffe expose paradoxalement la pudeur de sa désolation intime :

«Mes larmes se gonflaient dans leur source, mais ne jaillissaient point ; [...] Tous ces mouvemens, ces troubles, ces douleurs, s'agitaient confusément dans mon sein, mais sans éclater au dehors, comme ces volcans qui crevassent et déchirent les entrailles de la terre, et dont la surface est recouverte d'une riante pelouse. J'étais le consolateur universel, même beaucoup de gens me croyaient de la gaîté ; en effet je ne confiais guères ma tristesse qu'à un long corridor éclairé par une lampe sépulchrale, triste asyle de la mort, et où les prisonniers se promenant lentement semblaient s'accoutumer à la nuit et au silence des tombeaux ${ }^{73}$.

Plusieurs trouvent des ressources dans la fréquentation de soi et les charmes préromantiques de l'introspection. Mme Roland résiste :

« "Me voilà donc en prison", me dis-je. Ici je m'assieds et me recueille profondément. Je ne donnerai pas les moments qui suivirent pour ceux que d'autres estimeraient les plus doux de ma vie ; je ne perdrai jamais leur souvenir. Ils m'ont fait goûter, dans une situation critique, avec un avenir orageux, incertain, tout le prix de la force et de l'honnêteté dans la sincérité d'une bonne conscience et d'un grand courage [...] Je trouve que la prison produit sur moi à peu près les mêmes effets que la maladie; je ne suis

(72) Jean-Baptiste LOUVET, op. cit., p. 75, p. 91, p. 130, p. 131-132, p. 182-183.

(73) Honoré RIOUFFE, op.cit., p. 146. 
tenue, aussi, qu'à être là, et qu'est-ce que cela me coûte ? Ma compagnie n'est pas si mauvaise ».

Puis, éprouvée par sa deuxième arrestation, elle recherche des occupations intellectuelles :

« Je me trouvais dans cette disposition où toutes les impressions sont les plus vives et leurs effets alarmants pour la santé ; je me couchai sans pouvoir dormir ; il fallait bien rêver. [...] je me trouvai bien dupe d'accorder quelque chose à mes persécuteurs en me laissant froisser par l'injustice. [...] Ici comme à l'Abbaye, n'avais-je pas des livres, du temps ? N'étais-je plus moi-même ? [...] Je me propose d'employer les loisirs de ma captivité à retracer ce qui m'est personnel depuis ma plus tendre enfance jusqu'à ce moment ; c'est vivre une seconde fois que de revenir ainsi sur tous les pas de sa carrière, et qu'a-t-on de mieux à faire en prison que de transporter ailleurs son existence par une heureuse fiction ou par des souvenirs intéressants ? $\gg^{74}$.

Brissot affecte un détachement philosophique dans ce projet : «Je veux oublier un instant mon martyre et mettre à profit la solitude de ma prison $\gg^{75}$. Isnard qui se portraiture en stoïcien narre une expérience philosophique dont il paraît encore étonné :

«J'ai été grand dans mon infortune; mon âme s'est épurée dans le creuset du malheur [...] j'ai senti le bonheur naître de l'infortune, la nature a voulu que celle-ci eût ses utilités et même ses charmes [...]. Il m'est impossible d'exprimer quelles jouissances m'ont procuré ce silence, ce recueillement absolu, cette possession continuelle de ma pensée, cette étude suivie de mon être, ces fruits de sagesse et d'instruction que je sentais éclore en moi, cet abandon de la terre, ce lointain où j'appercevois et jugeois les criminelles folies des hommes, cette adoration sincère et croissante de la vertu, cette élévation intellectuelle vers les objets grands et sublimes et surtout, vers l'auteur de la nature, ce culte libre et pur que je lui adressois sans cesse $»^{76}$.

Il y a plus de légèreté dans le commerce bravache que Louvet dit a posteriori avoir entretenu avec la mort : «Je fis à part moi et mon traversin, quelques bons raisonnements sur les peines de la vie et les

(74) Mme Roland, op. cit., p. 65, p. 73, p. 292, p. 306

(75) Jean-Pierre BRISSOT, op. cit., t. 1, p. 24.

(76) Maximin ISNARD, op.cit., p. 20-23. 
douceurs de la mort ; elles ne pouvaient me fuir : je venais de m'assurer que l'opium et l'espingole étaient en bon état. Ainsi résigné, je m'endormis profondément ${ }^{77}$.

À la pensée de mourir, Mme Roland fléchit qui détourne Tacite : «La postérité rend à chacun sa place, mais c'est au temple de mémoire. Thémistocle n'en meurt pas moins en exil, Socrate dans sa prison, et Sylla dans son lit ». Elle se réconforte à l'espoir que ses Mémoires fourniront des sources pour l'histoire : «Mais l'histoire est là, elle tient ses burins, et prépare dans le silence la vengeance tardive des imitateurs de Barnevelt et Sidney $\gg^{78}$. Dans l'anéantissement qui le mène au suicide, Buzot se raccroche aux consolations de la postérité :

«C'est de moi seul que je veux ma récompense; c'est dans mon cœur, dans les souvenirs délicieux que j’y ai déposés [...], c'est là, dans ma conscience innocente et pure, que je trouve toute la force qui m'est nécessaire pour supporter [...] tout avec résignation, avec courage, jusqu'au moment où je pourrai me venger! Oui, me venger! venger mes amis, leur mémoire, de nos barbares oppresseurs ! [...] je ne vis que pour remplir cet unique et dernier devoir ! Et qui de nous, sans cet espoir consolateur, aurait pu consentir à trainer si longtemps une inutile et douloureuse vie, de district en district, de maison en maison, tantôt errant dans les landes sauvages ou dans les bois déserts de la Bretagne et du Périgord, tantôt parcourant deux cents lieues sur l'océan, exposés aux maladies, et aux tourments de la mer agitée, et aux incursions des Anglais piratant dans ces parages, et aux dangers, mille fois plus cruels que les Anglais et les orages, d'être rencontrés et reconnus par des Français, trouvant partout des cœurs froids, indifférents ou glacés par la peur, ou des âmes atroces altérées de notre sang et prêtes à nous livrer pour le plus modique intérêt ? Qui de nous, sans cet espoir consolateur, eût pu consentir à survivre à la liberté de notre pays, à la mort de nos amis, à notre indépendance personnelle ? Hélas ! Nous ne sommes déjà plus ! ou ce qui nous reste de nous-mêmes n'est plus qu'à la douleur ! [...] ! Vengeance, j'implore tes fiers et terribles accents ! Soutiens les restes languissants d'une vie consacrée désormais à te servir ${ }^{79}$.

Dans une victimisation thermidorienne et romantique, Riouffe se dit vivement affecté par le traumatisme de l'an II :

(77) Jean-Baptiste LouVET, op.cit., p. 172.

(78) Mme ROLAND, op. cit., p. 300-301.

(79) Charles ISNARD, op. cit., p. 96-97. 
« Misérable victime, jettée au milieu du monde social, il m'effraye et me navre. Je halète après la nature ; mais je suis, par rapport à elle, comme ces enfans qui, n'ayant point été nourris par leur mère, n'ont pas l'habitude du visage et du doux giron maternel, et ont laissé prendre le change à leurs affections filiales. Les révolutions des saisons, les variations des airs, les aspects lointains de la nature, le choc des élémens, tout retombe sur mon cœur. Le repos ne semble pas fait pour lui ; il est ouvert à toutes les atteintes, comme le serait un corps dépouillé de l'épiderme protectrice qui recouvre ses fibres, ses nerfs et ses muscles; de cette foule de sensations qui m'assiègent résulte nécessairement une plénitude de vie qui doit produire la satiété. Si vivre c'est sentir, quelle vie patriarchale égalerait la mienne, à peine arrivé cependant sur le seuil de l'âge viril $»^{80}$.

La relecture critique de ces Mémoires déconstruit l'histoire rêvée par les girondins d'une Révolution où ils n'occupent pas tout à fait la place qu'ils espéraient. Car la culture de ces hommes et femmes de la Gironde qui se revendiquent d'un héritage rousseauiste, affichent une sensibilité déiste et préromantique et conceptualisent la rupture révolutionnaire par la comparaison avec l'histoire des républiques antiques et de la révolution anglaise, ne les distingue finalement de leurs adversaires que - ce qui n'est pas rien - par leur hostilité à l'égard des aspirations populaires et le rejet affiché, quoique pas toujours effectif, de la violence. La relative parenté de leur culture politique, de leurs pratiques discursives, sinon de leurs desseins révolutionnaires, avec ceux de leurs vainqueurs, qui ajoute à la tragédie des expériences vécues en l'an II par ces républicains, explique en partie le succès de ces témoignages en ce XIX ${ }^{\mathrm{e}}$ siècle départageant mal mémoire, histoire, littérature et politique. En atteste ce vœu de Lamartine : « Pardonnons-nous donc, fils des combattants ou des victimes. Réconcilionsnous sur leurs tombeaux pour reprendre leur œuvre interrompue $! »^{81}$

Anne DE MATHAN

Maître de conférences d'histoire moderne Université de Bretagne occidentale anne.demathan@univ-brest.fr 